\title{
Development of cryogenically-cooled low noise amplifier for mobile base station receivers
}

\author{
ZHANG Xu ${ }^{1,2^{*}}$, LIU Jia ${ }^{2}$, CHEN ChuangYe ${ }^{2}$, LIU Wei ${ }^{2}$, LIU JingBin ${ }^{2}$, LI BaoHui ${ }^{1}$, HE Ming ${ }^{2}$, \\ ZHOU TieGe ${ }^{2}$, ZHAO XinJie ${ }^{2}$, YAN ShaoLin ${ }^{2} \&$ FANG Lan ${ }^{2}$ \\ ${ }^{1}$ School of Physics, Nankai University, Tianjin 300071, China; \\ ${ }^{2}$ College of Information Technical Science, Nankai University, Tianjin 300071, China
}

Received March 30, 2011; accepted August 18, 2011

\begin{abstract}
A new circuit model for designing and manufacturing an S-band low noise amplifier (LNA) with the software, Advanced Design System (ADS), is introduced in this paper. The proposed model involves shunted impedance at the grid to achieve a stable LNA without measuring the S-parameters of transistors at low temperatures. The LNA was measured over the operation band of 2.2-2.3 GHz, which has input and output standing wave ratios below 1.2. The noise figure of the manufactured LNA was about $0.2 \mathrm{~dB}$ and the gain was above $22 \mathrm{~dB}$, which indicated that our LNA worked well at $77 \mathrm{~K}$.
\end{abstract}

low noise amplifier, low temperature, gain, noise figure

Citation: Zhang X, Liu J, Chen C Y, et al. Development of cryogenically-cooled low noise amplifier for mobile base station receivers. Chinese Sci Bull, 2011, 56: 3884-3887, doi: 10.1007/s11434-011-4758-7

A cryogenic receiver front-end, comprising a high temperature superconducting filter and a cryogenically cooled low-noise amplifier (LNA), is an approach used to achieve high sensitivity and efficient frequency utilization for mobile base station receivers [1-3]. The LNA is one of the most critical components in receivers because it usually determines the sensitivity of the system. A cryogenic LNA with an even lower noise figure $(N F)$ is used in many applications such as radio astronomy, satellite communication and remote sensing. The $N F$ is one of the most important technological indicators because it determines the signal sensitivity of the whole system. The LNA is usually used as the first stage in receivers and its $N F$ adds to the whole system. To improve the sensitivity of the system, there is a growing demand for the design and manufacture of an LNA with high performance.

This paper describes how the LNA is designed using computer-aided software, ADS [4], a product of the Agilent Company. The DC-bias, stability and matching circuits are

\footnotetext{
*Corresponding author (email: nkzhangxu@ nankai.edu.cn)
}

all simulated. A new circuit model is proposed to minimize changes in the S-parameters of the transistors with environmental temperature. Through adding shunt impedance at the grid, the LNA can retain stable characteristics for all frequencies and at $77 \mathrm{~K}$. The PCB layout used to manufacture the LNA is generated to reflect the simulated circuit. The device is measured at room temperature and $77 \mathrm{~K}$ to obtain the $N F$, gain and voltage standing wave ratio (VSWR). The experimental results show that the LNA performs well at low temperatures and improves the sensitivity of the receivers.

\section{Circuit model and simulation}

In this study, the LNA was designed to work within the frequency range of 2.2-2.3 GHz. With a gain of over $22 \mathrm{~dB}$ and $N F$ at room temperature and $77 \mathrm{~K}$ lower than 0.8 and $0.3 \mathrm{~dB}$ respectively, both the input and output VSWR of the LNA should be below 1.2. To meet these requirements, the ATF-34143 of the Agilent Company was chosen for its high 
electron mobility transistor (HEMT). It has inherently low noise and high gain characteristics. It can work well at $77 \mathrm{~K}$ and has been used widely for cryogenically-cooled LNA designs.

On the basis of the properties of the ATF-34143, the DC-bias was chosen to be $3 \mathrm{~V}$ and $20 \mathrm{~mA}$. For this condition, the transistor has a $N F$ of $0.5 \mathrm{~dB}$, a gain of $17 \mathrm{~dB}$ and an output third-order intercept point (OIP3) of $30 \mathrm{dBm}$. A multi-stage configuration based on two ATF-34143 transistors was employed to achieve these demanding requirements. Usually, the ATF-34143 should work with a positive bias-voltage $V_{\mathrm{ds}}$ at the drain electrode and a negative bias-voltage $V_{\mathrm{gs}}$ at the grid electrode. However, if $V_{\mathrm{gs}}$ is supplied by a power source, the drain current $I_{\mathrm{ds}}$ will increase dramatically when the temperature falls to $77 \mathrm{~K}$. The variation in $I_{\mathrm{ds}}$ depresses the gain and increases the noise, resulting in degradation of the performance of the LNA.

To solve the problem and construct a high-performance LNA (at 77K) using the ATF-34143, a new circuit model, shown in Figure 1, is proposed. A resistor shunted by a capacitor is added to the source electrode as negative feedback. The source feedback will increase the negative bias-voltage at the grid electrode and maintain $V_{\mathrm{ds}}$ and $I_{\mathrm{ds}}$ constant even when the temperature changes. With reference to the alternating signal model [5] of the FET, shown in Figure 2, the input impedance can be calculated by:

$$
Z_{\text {in }}=R_{\text {in }}+j X_{\text {in }} \text {, }
$$

where

$$
\begin{gathered}
R_{\mathrm{in}}=-g_{m} \frac{1}{\omega^{2} C_{g s} C_{l s}}=-R_{1}, \\
X_{\text {in }}=-j\left(\frac{1}{\omega C_{g s}}+\frac{1}{\omega C_{l s}}\right)=-j X_{1} .
\end{gathered}
$$

It can be seen that the real and imaginary parts of the input impedance are both negative, which leads to the instability of the LNA at low frequency. Consequently, an impedance $Z_{g}=R_{g}+j X_{g}$ is shunted across the input and the input resistance shown in Figure 1 is then given by:

$$
\begin{aligned}
R_{\text {in }}^{\prime}= & \frac{R_{g} R_{\text {in }}\left(R_{g}+R_{\text {in }}\right)+X_{g}^{2} R_{\text {in }}+X_{\text {in }}^{2} R_{g}}{\left(R_{g}+R_{\text {in }}\right)^{2}+\left(X_{g}+X_{\text {in }}\right)^{2}} \\
= & \frac{R_{g} R_{1}\left(R_{1}-R_{g}\right)+X_{1}^{2} R_{g}-X_{g}^{2} R_{1}}{\left(R_{1}-R_{g}\right)^{2}+\left(X_{1}-X_{g}\right)^{2}} .
\end{aligned}
$$

The values of $R_{0}$ and $X_{0}$ need to be chosen correctly to make the real part $R_{\text {in }}{ }^{\prime}$ achieve a positive maximum and maintain the stable factor $K$ of the LNA over 1 among the frequencies $0-12 \mathrm{GHz}$, as shown in Figure 3. As a result of designing the new circuit model, the gain of the LNA at $77 \mathrm{~K}$ remains consistent at room temperature and the $N F$ decreases noticeably as will be seen in the experimental results. There is a major advantage in being able to design a cryogenically-cooled LNA without having to measure the $S$-parameters of the transistors at different temperatures.

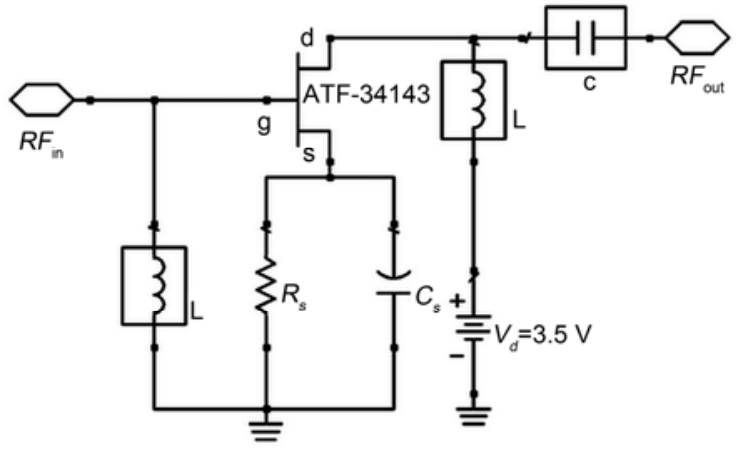

Figure 1 Input AC circuit model of the LNA.

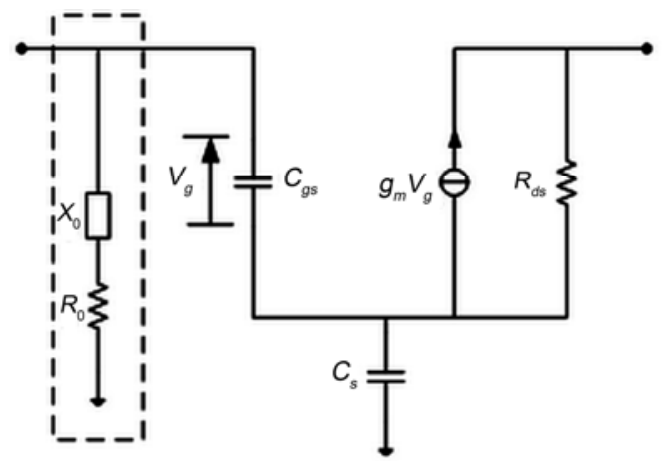

Figure 2 Equivalent input AC small signal circuit.

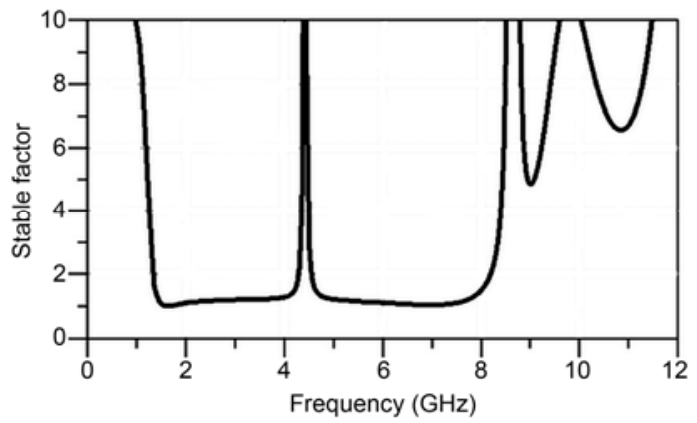

Figure 3 The curve of stable factor with the LNA at 0-12 GHz.

After designing the input and output matching circuitusing the L-microstrip network, the LNA was simulated using the electromagnetic full-wave analysis available in the layout space of the ADS software. The simulation results are shown in Figure 4 and Figure 5.

As shown in above figures, the NF of the LNA is below $0.55 \mathrm{~dB}$ and the gain is better than $25 \mathrm{~dB}$ for the $2 \mathrm{GHz}$ band. The input and output standing wave ratios of the LNA (not shown) are both smaller than 1.16. The simulation circuit was optimized repeatedly so that the LNA could work well at room temperature and $77 \mathrm{~K}$. The simulations are slightly better than the practical results because the welding 


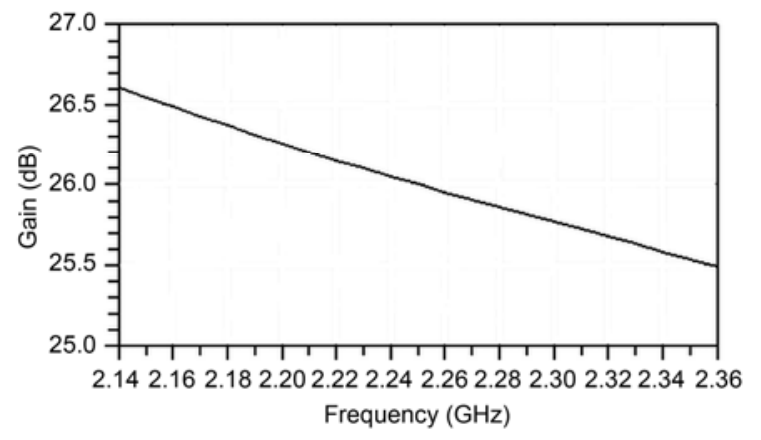

Figure 4 The simulated curve of gain versus frequency of the LNA.

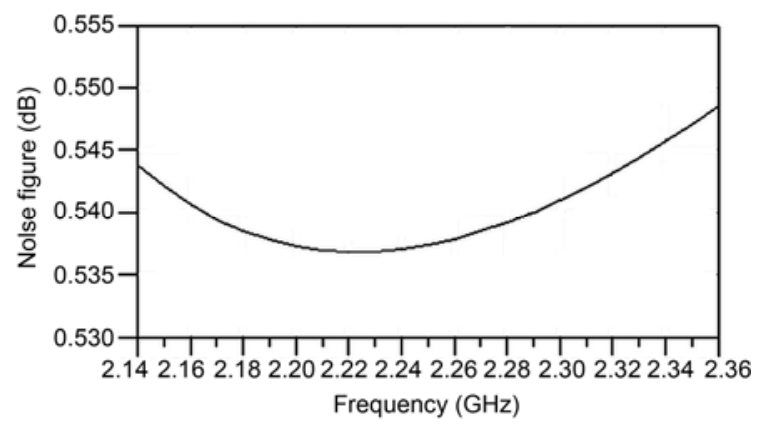

Figure 5 The simulated curve of $N F$ versus frequency of the LNA.

methods and the unmatched SMA joint can depress the actual performance of the LNA.

\section{Application examples of the ISV reduction}

Based on the simulated circuit, the layout of the LNA is designed automatically using the ADS software. The layout needs to be modified manually for convenient jointing and the addition of grounded holes. The devices, which include resistors, capacitors and transistors, are carefully welded onto the printed circuit schematic. Finally, an actual LNA is manufactured as shown in Figure 6.

The LNA performance is measured using a vector network analyzer, the AV3618, at room temperature and $77 \mathrm{~K}$. The gain of the LNA at $77 \mathrm{~K}$ is only about $1 \mathrm{~dB}$ higher than that at $300 \mathrm{~K}$ as shown in Figure 7. As a result of the new circuit design, the trans-conductance of the transistor does not degrade at cryogenic temperatures. The slight increase of gain at $77 \mathrm{~K}$ is due to the increased electron mobility at low temperatures. The curves of the $N F$ versus frequency at the two temperatures are shown in Figure 8 . At $77 \mathrm{~K}$, the LNA has a noise figure of $0.176 \mathrm{~dB}$, namely the equivalent noise temperature is $12.4 \mathrm{~K}$. The $N F$ at a liquid nitrogen temperature obviously decreases by about $0.6 \mathrm{~dB}$. Two possible reasons for this include the reduction of the resistor values in the LNA at $77 \mathrm{~K}$ and the increasing electron mobility ratio of the ATF-34143.

To verify our new circuit model for improving the

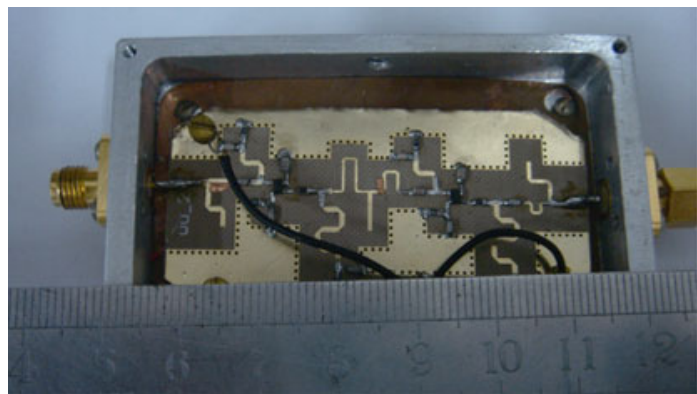

Figure 6 The photograph of the manufactured LNA.

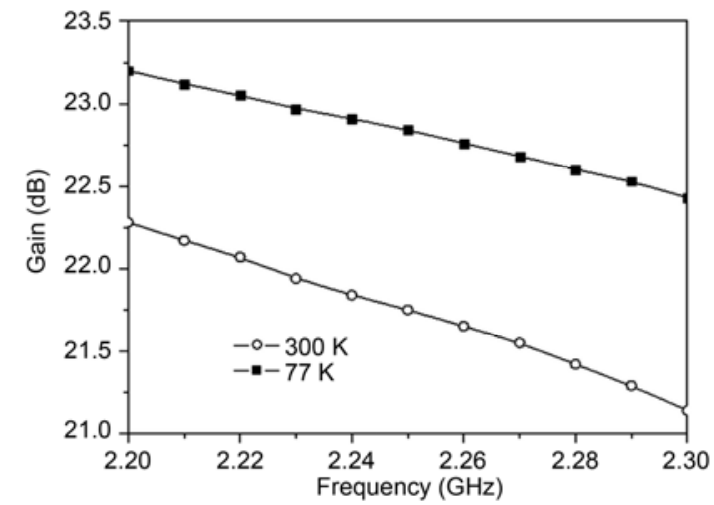

Figure 7 The experimental curve of gain versus frequency at 77 and $300 \mathrm{~K}$.

stability of the LNA, the $S$-parameters and $N F$ versus temperature are measured at $2.15 \mathrm{GHz}$ as shown in Figure 9 and Figure 10. On one hand, there is only a little change on in the $S$-parameters, including $S_{11}, S_{21}$ and $S_{22}$, at different temperatures because our new circuit model maintains the stability of the LNA. The slight temperature dependence of the $S$-parameters is very useful when designing cryogenically-cooled LNA with computer-aided software. On the other hand, the $N F$ visibly decreases with decreasing temperature, which is required for a cooled LNA. The extremely low $N F$ (about $0.18 \mathrm{~dB}$ ) of LNA at low temperatures will improve the sensitivity performance for mobile base station receivers comprising a HTS filter and a cryogenic LNA.

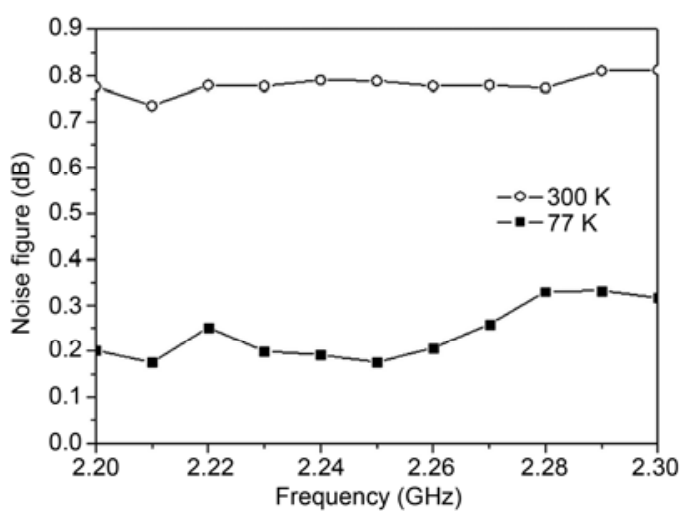

Figure 8 The experimental curve of $N F$ versus frequency at 77 and $300 \mathrm{~K}$. 


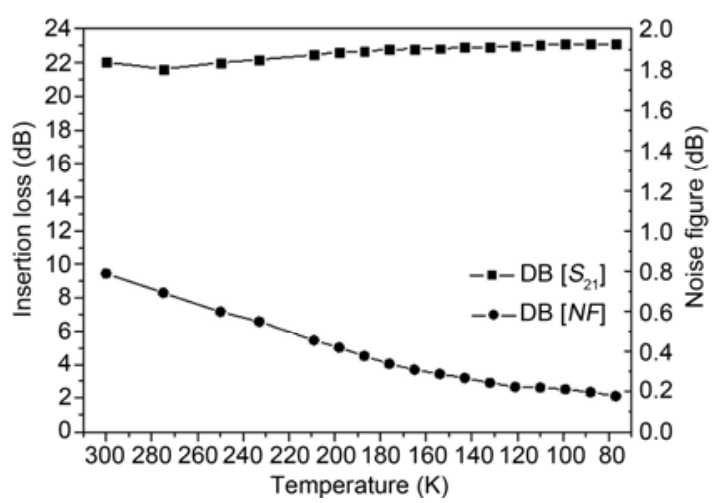

Figure 9 The curves of $S_{21}$ and $N F$ versus temperature at $2.15 \mathrm{GHz}$.

\section{Conclusions}

This paper presents a new circuit model for preventing the changes in the $S$-parameter characteristics of transistors with changes in environmental temperature. It enables the convenient design and then manufacture of a cryogenicallycooled LNA without measuring the $S$-parameters of the transistors at different temperatures. The LNA was measured in the operation band of 2.2-2.3 GHz, which had a $N F$ of $0.176 \mathrm{~dB}$ and a gain of $22.8 \mathrm{~dB}$ at the central frequency. The experimental results confirmed that there was only a slight change in the $S$-parameters, including $S_{11}, S_{21}$ and $S_{22}$, between room temperature and $77 \mathrm{~K}$. The high-performance LNA, designed using our new circuit model, enables further improvements in the sensitivity of mobile base station receivers.

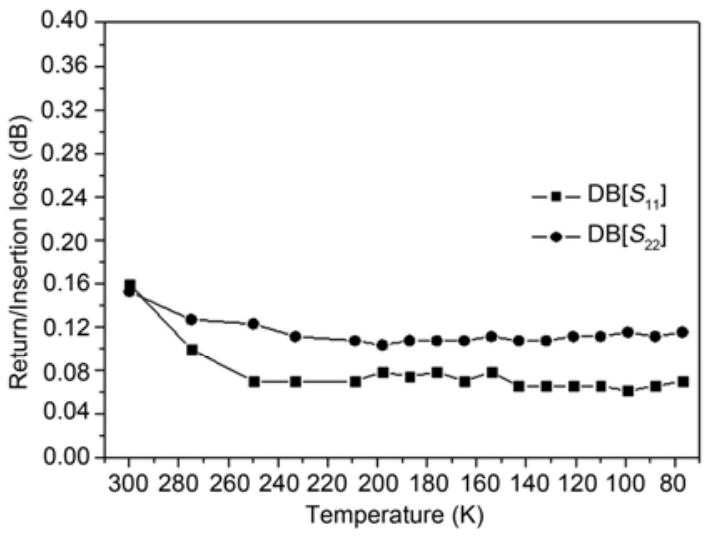

Figure 10 The curves of $S_{11}$ and $S_{22}$ versus temperature at $2.15 \mathrm{GHz}$.

This work was supported by the Specialized Research Fund for Doctoral Program of Higher Education in China (200800551009) and the National High Technology Research and Development Program of China (2009AA03Z208).

1 Klauda M, Kaesser T, Mayer B, et al. Superconductors and cryogenics for future communication systems. IEEE Trans Microw Theory Tech, 2000, 48: 1227-1239

2 Zhou C X, Xia H H, Zuo T, et al. Development of X-band high temperature superconducting filters. Chinese Sci Bull, 2010, 55: 168-171

3 Ning J S, Yang K, Luo Z X, et al. Design of high temperature superconducting contiguous-band diplexer. Chinese Sci Bull, 2009, 54: 1279-1281

4 Bretchko P, Ludwig R. RF Circuit Design: Theory \& Applications. 2nd ed. New Jersey: Prentice Hall Press, 2007

5 Robert E C. Foundations for Microwave Engineering. New York: John Wiley \& Sons Publication, 1965

Open Access This article is distributed under the terms of the Creative Commons Attribution License which permits any use, distribution, and reproduction in any medium, provided the original author(s) and source are credited. 